\title{
A GESTÃO DO CONHECIMENTO NA CULTURA MAKER: OS ACHADOS DE UMA PESQUISA EXPLORATÓRIA EM UM HACKERSPACE
}

Steffi Becker ${ }^{1}$, Nelson Tenório²

'UniCesumar, Programa de Pós-Graduação Stricto Sensu em Gestão do Conhecimento nas Organizações, Maringá, PR. E-mail: steffiabecker@gmail.com

${ }^{2}$ Instituto Cesumar de Ciência, Tecnologia e Inovação (ICETI), Maringá, PR. UniCesumar, Docente permanente do Programa de Pós-Graduação Stricto Sensu em Gestão do Conhecimento nas Organizações. E-mail: nelson.tenorio@unicesumar.edu.br

\section{RESUMO}

Com a crescente da cultura maker tem surgido diversos espaços visando a inovação tecnológica por meio da interação social, como os hackerspaces. Nesse sentido, o objetivo deste artigo é compreender como a Gestão do Conhecimento (GC) pode apoiar os hackerspaces para que o conhecimento de seus membros se torne acessível aos seus novos integrantes. Nesse sentido, foi realizada uma pesquisa bibliográfica e exploratória por meio de conversas informais com membros de um hackerspace localizado no estado do Paraná. Finalmente, os achados desta pesquisa apontam que a GC apoia os hackerspaces na criação de bases de conhecimento, no compartilhamento do conhecimento entre seus membros e novos integrantes, bem como no estímulo à criação do conhecimento.

Palavras-chave: espaço maker, modelo $\mathrm{SECl}$, acesso ao conhecimento, trabalho colaborativo, aprendizado.

\section{THE MANAGEMENT OF KNOWLEDGE IN CULTURE MANUFACTURER: THE FINDINGS OF AN EXPLORATORY RESEARCH IN A HACKERSPACE}

\begin{abstract}
With the growing culture of the maker, several spaces raise in order to promote technological innovation through social interaction, such as hackerspaces. In this sense, this paper is to understand how the Knowledge Management can support hackerspaces so that the knowledge of its members becomes accessible to new members. To accomplish the research goal we carried out bibliographic and exploratory study through informal conversation with three members of a hackerspace located in the state of Paraná. Finally, the findings of this research point out that the CG supports hackerspaces in the creation of knowledge bases, in the sharing of knowledge among its members and new members, and in stimulating the creation of knowledge.
\end{abstract}

Keywords: makerspace, SECI model, knowledge access, collaborative work, learning.

\section{INTRODUÇÃO}

O mundo contemporâneo é caracterizado por ter uma economia altamente globalizada e dinâmica, onde os avanços em produtos tecnológicos se tornam essenciais para a consolidação econômica no mercado. Esse contexto $_{2}$ dinâmico e interconectado, vem transformando o modelo da economia e seus modos de produção, de comunicação e de trocas de informações. Nesse novo modelo, denominado por Houghton e Sheehan (2000) como 'Economia do Conhecimento' ou de acordo com Cavalcanti, Gomes e Pereira (2001) a 'Sociedade do Conhecimento', em que a evolução tecnológica é constante e cada vez mais acelerada, o conhecimento se tornou um recurso essencial para as pessoas e para organizações, sendo considerado por Martinez et al. (2007) um recuso que proporciona a criação de produtos e serviços os quais tornam as nações mais competitivas.

Estudos relativos a relevância do conhecimento para as organizações não são pautas atuais, haja vista que pesquisas 
relacionadas ao tema vêm sendo realizadas a mais de vinte anos. Desse modo, faz-se necessária a gestão do conhecimento (GC) para auxiliar as organizações obterem vantagens competitivas e sustentabilidade no mercado em que atuam (NONAKA; TAKEUCHI, 1997). Entretanto, é nos últimos anos que a GC vem ganhando força, diante do irreversível desenvolvimento tecnológico mostrando-se relevante para a ciência.

Diante desse cenário, onde a tecnologia exerce papel fundamental, há um forte movimento para o surgimento de organizações voltadas para a criação e difusão de conhecimento tecnológico, como os makerspace, hackerspaces ou FabLabs. Os hackerspaces são organizações sem fins lucrativos voltadas a criação de inovações, tendo como objetivo a 'inovação pela inovação'. Tratam-se de laboratórios comunitário e cooperativo que difundem a aprendizagem, principalmente na área de inovação e que buscam geram resultados para a sociedade onde essa organização está inserida (BJØRN et al., 2017). Entretanto, esses espaços dependem do conhecimento tácito das pessoas, não deixando claro como esse conhecimento flui entre os seus membros, principalmente para àqueles recém-chegados. Portanto, investigar meios de gerenciar o conhecimento existente nos hackerspaces pode amplificar a sua audiência tornando-os mais democráticos e acessível para as pessoas.

Considerado a relevância e o impacto dessas organizações na sociedade atual, este artigo tem por objetivo compreender como a GC pode apoiar os hackerspaces para que o conhecimento de seus membros se torne acessível para novos integrantes. Este trabalho está organizado em cinco seções. A primeira seção debate a cultura maker e os hackerspaces seguida dos conceitos da gestão do conhecimento para o embasamento teórico da presente pesquisa. Na sequência é apresentada a metodologia, em que se mostra os delineamentos utilizados para se alcançar os resultados deste trabalho. A seção de resultados e discussão apresenta um diálogo entre a teoria e os resultados alcançados com conversas informais com membros de um hacker hackerspace. Por fim, apresenta-se a conclusão da pesquisa e seus direcionamentos futuros, seguida das referências bibliográficas.

\section{A CULTURA MAKER E OS HACKERSPACES}

$O$ conceito de maker é relativamente novo e aplicado para descrever a crescente atenção dada às construções de espaços de atividades que permitem a aprendizagem de forma divertida e envolvente. Na cultura maker há um processo onde uma pessoa pode se tornar um criador de novas 'coisas' (GIANNAKOS; DIVITINI; IVERSEN, 2017). Esses espaços, físicos ou digitais, permitem aos participantes um ambiente propício à aprendizagem cooperativa e colaborativa (BJ $\varnothing \mathrm{RN}$ et al., 2017) que por sua vez, possibilita a resolução de problemas tecnológicos, tornando propício o surgimento de novos inovadores produtos. A grande maioria dos espaços considerados pertencentes à cultura maker, possuem enfoque na comunidade global para a inovação. Assim, suas atividades comportam designers, engenheiros em diversas áreas, artistas, programadores e estudiosos de outras áreas que estimulam a capacidade dos futuros inventores, inovadores e pessoas que querem mudar e melhorar o mundo em que vivem (GIANNAKOS; DIVITINI; IVERSEN, 2017). Esses locais, quando bem planejados, proporcionam um ambiente adequado para a aprendizagem e crescimento educacional. Nesses ambientes comunitários os jovens podem se beneficiar de diversas oportunidades que propiciam oportunidades para experimentar uma forma envolvente, alegre e pedagogicamente adequada de aprendizagem. Exemplos desses ambientes são os hackerspaces, makerspaces, TechShops e Fablabs.

O termo hackerspace é a junção das palavras hacker, sinônimo de adaptar objetos por meio do uso de tecnologias, e space, que significa um espaço ou ambiente físico. Esses espaços, também são conhecidos como hacklab, makerspace ou creative space, e possuem o formato de laboratórios comunitários que visam agregar, convergir e inspirar invenções que beneficiem a sociedade (HARNETT; TRETTER; PHILIPP, 2014). Sendo assim, os hackerspaces podem ser caracterizados como laboratórios abertos com atividades executada nos moldes de workshop e operados democraticamente pelos seus membros, que se autocaracterizam como uma comunidade amistosa de pensadores.

Nos hackerspaces as pessoas com interesses em comum, normalmente voltados para as áreas de ciência, tecnologia ou eletrônica, podem se encontrar e aprender de forma colaborativa. Nesse sentido, os 
hackerspaces, em seu cerne, estimulam a socialização e a colaboração entre as pessoas envolvidas. Portanto, essas organizações podem ser vistas sob a perspectiva de laboratório comunitário, oficina-garagem ou estúdio, onde as pessoas de diversas áreas podem trocar experiências e conhecimento para construírem juntas soluções para determinados problemas de diferentes aspectos, incluindo, em sua grande parte, $\mathrm{o}$ aspecto social. Esses ambientes que, por si só, possibilitam a aprendizagem entre pares fornecem insights interessantes para invenções diversas (BJØRN et al., 2017). Além disso, esses espaços reúnem as pessoas para gerar novas ideias e trabalhar em protótipos conceituais de novos produtos socialmente e globalmente relevantes e inovadores (GIANNAKOS; DIVITINI; IVERSEN, 2017). Portanto, os ambientes da cultura maker, e em particular os hackerspaces, alicerçam-se fortemente na aprendizagem colaborativa, onde os seus membros, que possuem diferentes perfis, habilidades e competências, são estimulados a aprenderem continuamente uns com os outros. Esse princípio de troca de conhecimentos vai ao encontro dos fundamentos que alicerçam a GC.

\section{A GESTÃO DO CONHECIMENTO}

O conhecimento é um fator estratégico que contribui para que uma organização se perpetue ao longo do tempo. Considerando a relevância do tema, surge a GC com o intuito de aprimorar a compreensão dos tipos de conhecimento e como eles surgem para sugerir áreas do conhecimento distintas, proporcionando a maximização da aprendizagem (ABREU; ABREU; PRADA, 2013). De acordo com Strauhs (2012), o papel da GC nas organizações é proporcionar condições para criar, adquirir, organizar e processar informações estratégicas gerando benefícios que proporcionem o aumento da competitividade. Desse modo, a GC propõe alguns ciclos para a criação do conhecimento, como àqueles propostos por Bukowitz e Willians (1999), Meyer e Zack (1996), Wiig (1993), e o mais conhecido deles, o modelo $\mathrm{SECl}$ (Socialização, Externalização, Combinação e Internalização) ou 'espiral do conhecimento' de Nonaka e Takeushi (1997). Tais modelos são importantes porque servem como direcionadores da GC dentro das organizações (DALKIR, 2011). 0 modelo $\mathrm{SECl}$, por exemplo, propõe a união entre o conhecimento tácito e o explicito, e supõe que a junção desses dois tipos de conhecimentos gera um novo conhecimento. Dessa forma, o SECl é dividido em quatro quadrantes, denominados de socialização, externalização, combinação e internalização.

A socialização do conhecimento ocorre por meio da interação entre as pessoas que passam a compartilhar seus conhecimentos, suas habilidades, experiências, ideias e percepções. Por sua vez, a internalização faz com que a pessoa expresse o seu conhecimento em uma linguagem escrita/representativa. A combinação é o processo pelo qual o conhecimento explícito é combinado por meio da troca de documentos, reuniões, conversas ao telefone ou redes de comunicação computadorizada. Por fim, a internalização sugere que a pessoa, agora munida de um novo conhecimento, aplique-o no seu dia a dia para resolver problemas e propor inovação. Portanto, a etapa de combinação da espiral do conhecimento é a responsável por possibilitar a criação de um novo conhecimento, que pode ser inovador, e a partir do momento que há uma combinação de conhecimentos e ele é aplicado a uma determinada atividade e internalizado pelas pessoas (NONAKA; TAKEUCHI, 1997). Esses dois momentos estão fortemente relacionados com o 'aprender fazendo', o que é a proposta principal dos ambientes de cultura maker.

\section{METODOLOGIA}

A presente pesquisa caracteriza-se por ter objetivo exploratório de abordagem qualitativa fazendo o uso de procedimentos bibliográfico e de campo (CRESWELL, 2013), realizadas em duas fases. A primeira fase foi caracterizada por um levantamento da literatura para a compreensão do tema deste artigo. Nessa fase foram realizadas buscas nas bases de dados on-line Emerald Insight, ACM Digital Library, Science Direct e o Portal de periódicos da CAPES, utilizando-se as seguintes palavras-chave em Língua Portuguesa nos campos título e resumo: 'cultura maker' ou 'espaço maker' ou 'hackerspace' ou 'FabLab'. Igualmente, foram pesquisadas as seguintes palavras chaves em Língua Inglesa nos campos title e abstract: 'maker culture' ou 'makerspace' ou 'hackerspace' ou 'FabLab'. Além disso, delimitou-se a pesquisa a artigos completos publicados a partir de 2013. $\mathrm{Na}$ segunda fase, foram conduzidas três conversas informais, uma delas durante uma das duas visitas para observação do campo e as demais por meio do uso de um aplicativo via 
smartphone. As conversas foram conduzidas com membros ativos e fundadores de um hackerspace localizado na cidade de Maringá, no noroeste do estado do Paraná, durante o mês de julho de dois mil e dezoito. A primeira conversa foi conduzida no hackerspace com um de seus fundadores e tendo a duração de, aproximadamente, vinte minutos. Por questões de conflitos de agenda, as outras duas conversas foram conduzidas por meio do aplicativo de bate-papo WhatsApp, em que cada conversa teve duração média de quinze minutos. Os dados coletados foram analisados empiricamente com base em anotações feitas durante as conversas informais.

\section{RESULTADOS E DISCUSSÃO}

Favorecer a expansão dos hackerspaces para pessoas dos mais diferentes perfis, significa aumentar a diversidade de inovação tecnológica nesses ambientes, aprimorando assim 0 conhecimento dessas organizações. Para que isso ocorra, faz-se necessário que o conhecimento de seus membros esteja acessível aos novos integrantes. Nesse sentido, apresenta-se a seguir três achados encontrados durante as conversas informais com os membros do hackerspace em questão.

Registro das atividades. Durante uma das visitas, em conversa informal, foi perguntado a um dos fundadores do hackerspace, que estava presente no momento da visita, como o conhecimento era compartilhado entre os seus membros, ele observou que as atividades realizadas no hackerspace estão embasadas na troca de conhecimento e cooperação entre os seus participantes, e isso se dá de modo tácito, por meio da comunicação e da colaboração entre os seus membros, que possuem diferentes formações acadêmicas. A ideia do ambiente é promover a fluência do conhecimento por meio da comunicação para que esses se ajudem mutuamente. Entretanto, existe uma documentação formal, que registra algumas das atividades realizadas no local. Essa documentação se encontra em forma de materiais de treinamento e documentação de projetos, mas esses ficam em posse de seus membros, que não costumam disponibilizá-los em um local compartilhado. O mesmo ainda ressaltou que no hackerspace 'as coisas simplesmente acontecem', como ocorre em outros hackerspaces do mundo, e assim, os projetos são realizados e seus membros aprendem fazendo. Nesse sentido, nota-se que a fonte de conhecimento dos hackerspaces é caracterizado pelo conhecimento tácito, i.e., aquele que é inerente às pessoas (NONAKA; TAKEUCHI, 1997). Embora o hackerspace não dê a devida atenção ao conhecimento explícito, uma vez que é fortemente baseado na cultura maker, a saída de algum de seus membros pode comprometer o conhecimento do grupo. Nesse sentido, é importante que o conhecimento contido nas atividades da organização seja registrado em uma base de conhecimento compartilhada para que não se perca, e assim, seus membros possam acessar esse conhecimento quando for necessário. Desse modo, a GC oferece diferentes ciclos, processos, modelos e ferramentas, como aqueles sugeridos por Bukowitz e Willians (1999) Meyer e Zack (1996), Nonaka e Takeuchi (1997) e Wiig (1993), que permitem traçar estratégias para o registro de atividades e a criação de uma base de conhecimento acessível (DALKIR, 2011). Logo, esse primeiro achado requer a necessidade do registro das atividades do hackerspace para o acesso ao conhecimento explícito por parte de seus membros e novos integrantes, formando assim uma base de conhecimento contínua.

Compartilhamento do conhecimento por meio de cursos e mentorias. Um outro membro, uma jovem que cursa a graduação em letras português-inglês, quando questionada como se interessou pelo hackerspace, relatou que, apesar de ser da área de humanas, é uma apaixonada por tecnologia e, portanto, entrou como membro do hackerspace para desenvolver um projeto que usa a tecnologia para solucionar problemas de comunicação entre as pessoas e possibilita traduções de textos que considerem, não apenas as palavras, mas os seus significados (i.e., semântica). Portanto, ela participa ativamente de cursos e palestras de tecnologias ministrada por outros membros do hackerspace, que são gratuitos e abertos à comunidade. Nesse caso a GC, que possui caráter multidisciplinar, favorece e promove a integração entre diferentes áreas do conhecimento, proporcionando a maximização da aprendizagem (ABREU; ABREU; PRADA, 2013). Dessa forma, o segundo achado mostra que compartilhar o conhecimento por meio de cursos e mentorias favorece o nivelamento técnico entre as pessoas, principalmente os novos integrantes.

Criação do conhecimento. Finalmente, um terceiro membro, um estudante que está finalizando a faculdade de Ciências Biológicas e 
frequenta o ambiente do hackerspace diariamente, relatou que a sua experiência no hackerspace cria condições para ele desenvolver o projeto de uma estufa que permita o cultivo de mudas características de determinadas regiões do mundo. De acordo o estudante, o hackerspace é fundamental para o desenvolvimento de seu projeto uma vez que 'várias pessoas viram a ideia e foram dando dicas e ajudando a pensar em como fazê-la'. Dessa forma, observa-se aqui a criação do conhecimento, resultado da combinação do modelo SECl (NONAKA; TAKEUCHI, 1997), pois o conhecimento tácito desse estudante foi combinado com 0 conhecimento de outros membros do hackerspace. Portanto, o último achado é que os hackerspaces promovem a criação do conhecimento por meio da combinação, conforme sugere o modelo SECl.

Dessa forma, a GC pode aumentar a audiência dos hackerspaces e a sua democratização por meio do registro das suas atividades, tornando o conhecimento explícito; do compartilhamento do conhecimento, ofertando cursos gratuitos; e, finalmente, potencializando a criação do conhecimento por meio da democratização e do ingresso de novos participantes.

\section{CONCLUSÃO}

Este artigo teve como objetivo compreender o modo em que a GC pode apoiar os hackerspaces para que o conhecimento de seus membros se torne acessível para novos integrantes. A pesquisa, bibliográfica e exploratória, apresentou três achados que apontaram que a GC apoia os hackerspaces por meio da criação de bases de conhecimento, do compartilhamento do conhecimento entre seus membros e novos integrantes e do estímulo à criação de novos conhecimentos. Quanto ao primeiro achado, observou-se eu, até o momento, os hackerspace não possuem ferramentas ou métodos de armazenagem do conhecimento nele criado, o que compromete a aprendizagem dos integrantes que futuramente participarão do hackerspace, isso pois, esses não terão contato direto com o outrora criador de um determinado conhecimento importante para a organização. Quanto ao segundo achado, mostra que 'compartilhar o conhecimento por meio de cursos e treinamentos' favorece o nivelamento técnico entre as pessoas e a disseminação do conhecimento. Unindo o primeiro e o segundo achados desta pesquisa constatou-se que a combinação entre os diversos conhecimentos conforme sugere o modelo $\mathrm{SECl}$, 'promovem a criação do conhecimento'. Ressalta-se que esse processo se torna muito mais efetivo e disseminável quando há o registro dessas atividades. Esses conhecimentos registrados e armazenados fornecerão base para os novos membros em seus processos de criação de novos conhecimentos. Nesse sentido a GC pode contribuir para o aperfeiçoamento das atividades nesse espaço.

\section{REFÊRENCIAS}

ABREU, A. F.; ABREU, F. P.; PRADA, C. A. Gestão do Conhecimento. Florianópolis: SENAI, SC, 2013.

BUKOWITZ, W R.; WILLIAMS, R L. The knowledge management fieldbook. Financial Times Prentice Hall, 1999.

CAVALCANTI, M. C. B.; GOMES, E B. P.; PEREIRA A. F. A gestão de empresas na sociedade do conhecimento: um roteiro para a ação. Rio de Janeiro: Campus, 2001.

CRESWELL, J. W. Research design: qualitative, quantitative, and mixed methods approaches. Thousand Oaks: Sage publications, 2013.

DALKIR, K. Knowledge management in theory and practice. 2.ed. England: The MIT Press, 2011.

GIANNAKOS M. N.; DIVITINI M.; IVERSEN O. S. Entertainment, engagement, and education: Foundations and developments. IN digital and physical spaces to support learning through making. Entertainment Computing, v. 21, p. 7781, Jun. 2017.

HARNETT, C. K.; TRETTER, T. R.; PHILIPP, S. B. Hackerspaces and engineering education. In: Frontiers In education conference (FIE), Proceedings... Madrid, Spain: IEEE, 2014 p. 1-8.

HOUGHTON, J.; SHEEHAN, P. A Primer on the knowledge management. Centre for Strategic Economic Studies. Victoria University, Melbourne, 2000.

LUNDBJERG H. E.; PFLUG VON DER, O. J.; KANTO, R.; BJØRN, P. The Hackerspace manifested as a DIY-IoT Entity. In: of 15th European conference on computer-supported cooperative work - 
exploratory papers. 15., Proceedings... Sheffield, Reino Unido, 2017. p. 205-221.

MARTÍNEZ, M.; PRIETO A. T.; RINCÓN, Y.; CARBONELL, D. Aprendizaje en las comunidades de conocimiento desde uma perspectiva organizacional: una aproximación teórica. ORBIS Revista Científica Ciencias Humanas, v. 3, n. 7, p. 46-64, 2007.

MEYER, M.; ZACK, M. The design and implementation of information products. Sloan Management Review, v. 37, n. 3, p. 43-59, 1996.

NONAKA, I.; TAKEUCHI, $H$. Criação de Conhecimento na Empresa: como as empresas japonesas geram a dinâmica da inovação. Rio de Janeiro: Campus, 1997.

STRAUHS, F. R. Gestão do conhecimento nas organizações. Curitiba: Aymará Educação, 2012.

WIIG, K. Knowledge management foundations. Arlington, TX: Schema Press, 1993.

Recebido para publicação em 17/08/2018

Revisado em 02/09/2018

Aceito em 06/09/2018 